\title{
Rapid Spread of COVID-19! What Are the Cases of COVID-19 Infection in Dental Clinics in Korea?
}

\author{
Ji-Hyoung $\operatorname{Han}^{\dagger}$ \\ Department of Dental Hygiene, Suwon Science College, Hwaseong 18516, Korea
}

As of August 2020, the number of coronavirus disease 2019 (COVID-19) cases around the world was about 22 million and the number of deaths was about $770,000^{1)}$. The virus was first discovered in China and it spread to South Korea, Europe, and the United States. Currently, it is prevalent in the Americas, India, and the Middle East as well.

COVID-19 is transmitted through air droplets. South Korea has been successful in controlling the spread of infection using a strict quarantine system and public health awareness on measures such as hand washing and the wearing of masks. However, following the group infections in Shincheonji, Itaewon, and recently the Sarangjeil church, South Korea has active cases. Although dental clinics in Korea generate significant aerosol that may facilitate droplet infections, they have recorded no cases ${ }^{2}$. There are rare cases in Korea, and asymptomatic patients may have visited dentists; however, no case has been reported, and this may be attributed to the effectiveness of the wearing of masks and gloves and infection control in dental clinics.

In Korea, the Dental Infection Control Standard Policy \& Procedure ${ }^{3)}$ established infection control systems classified into: (1) standard precautions/occupational safety; (2) instrument reprocessing; (3) environmental and waterline management of dental institutions; (4) special infection control, which covers guidelines on infection control and precautions such as hand washing and the wearing of personal protective equipment. The current status of infection control in dental clinics may provide insights into solutions for preventing COVID-19 infections. With the increasing prevalence of severe viral diseases such as COVID-19, thorough infection control and continued research is paramount.

\section{References}

1. Retrieved August 17, 2020, from https://search.naver.com/ search.naver?sm=top_sug.pre $\& \mathrm{fbm}=1 \&$ acr $=1 \&$ acq $=\% \mathrm{EC} \%$ 84\%B8\%ЕA\%B3\%84\%ЕC\%BD\%94\%ЕB\%A1\%9C\%ЕB\% $82 \% 98 \& q d t=0 \& i e=u t f \& q u e r y=\% \mathrm{EC} \% \mathrm{~A} 0 \% 84 \% \mathrm{EC} \% 84 \%$ B8\%ЕA\%B3\% 84+\%ЕC\%BD\%94\%ЕB\%A1\%9C\%ЕB\%82 \%98+\%ED\%99\%95\%ЕC\%A7\%84\%ЕC\%9E\% 90

2. Retrieved August 16, 2020, from http://www.dentalarirang. com/news/articleView.html?idxno=29396.

3. Korea Dental Association: Dental infection control standard policy \& procedure. Ministry of Health \& Welfare, Sejong, pp.19-236, 2020. 